Mendez Garrido, J.M. \& Conde Vélez, S. (2018). La autorreflexión inicial: una estrategia para la mejora de la práctica docente. Revista Electrónica Interuniversitaria de Formación del Profesorado, 21(1), 17-31.

DOI: http://dx.doi.org/10.6018/reifop.21.1.270591

\title{
La autorreflexión inicial: una estrategia para la mejora de la práctica docente
}

Juan Manuel Méndez Garrido, Sara Conde Vélez

Universidad de Huelva, España

\section{Resumen}

Este artículo presenta un estudio cuyo objetivo principal es conocer las reflexiones del alumnado al inicio del curso para mejorar la práctica docente del profesorado universitario a través de la autorreflexión inicial. El cuestionario se convierte en un documento de autorreflexión inicial explorando las valoraciones que los estudiantes hacen al respecto. Para ello, se aplica un cuestionario de preguntas abiertas a los estudiantes de la asignatura de Métodos de Investigación y Diagnóstico en Educación Social, en el que se reflexiona sobre los siguientes aspectos: situación inicial, punto de partida, ideas previas a la realización del curso; reflexión sobre el proceso de trabajo; reflexiones sobre la actitud del profesorado y otras sugerencias.

Para el tratamiento de los datos se hace un análisis cualitativo, organizando la información obtenida en las distintas categorías establecidas. De las aportaciones obtenidas en cada uno de los apartados se seleccionarán los elementos comunes expresándolos con una frase e incluyendo entre paréntesis el número de veces que se repite. En la valoración de los objetivos se sumarán los valores obtenidos por cada uno de ellos. Los resultados revelan la autorreflexión inicial como una útil herramienta, para el profesorado, que puede ser tenida en cuenta para el diseño de las clases.

\section{Palabras clave}

Autorreflexión inicial; formación del profesorado; práctica docente; enseñanza universitaria.

\section{Contacto:}

Sara Conde Vélez: Dirección postal completa. Dirección postal completa: Universidad de Huelva. Departamento de Educación. Campus “El Carmen”, 21771, Huelva, España. Correo-e: sara.conde@dedu.uhu.es 


\title{
Initial self-reflection: a strategy for the improvement of the teaching practice
}

\begin{abstract}
This article presents a study whose main objective is to know the reflections of students at the beginning of the course to improve the teaching practice of university teachers through the initial self - reflection. The questionnaire becomes an initial self-reflection document exploring the assessments students make about it.
\end{abstract}

To do this, a questionnaire is applied to open-ended questions to students of the subject of Research Methods and Diagnosis in Social Education, which reflects on the following applies ; reflection on the work process; reflections on the attitude of teachers and other suggestions.

For the treatment of qualitative data analysis is done by organizing the information obtained in the different categories established. The contributions obtained in each of the sections the common elements will be selected by expressing a parenthetical phrase and including the number of times it is repeated. In assessing the objectives values obtained by each of them. The results reveal the initial self-reflection as a useful tool for teachers, which can be taken into account in the design of the classes will be added

\section{Key words}

Initial self-reflection; teacher training; teaching practice; university education.

\section{Introducción}

Partir de las ideas previas del alumnado a atender en nuestras aulas es una tarea común en el profesorado actual. En estudios anteriores sobre este tema (Ávila y Conde, 2013) se comentaba que con este fin, a principios del año académico, en cualquier curso, se prepara un documento de trabajo para el alumnado que asiste a la asignatura de Tutoría y Orientación en Educación Infantil. Se denomina el documento "Autorreflexión Inicial" en el que se propone una reflexión para que se valore de manera personal las expectativas del curso que se va a compartir, indicando que las aportaciones serán tenidas en cuenta para la mejora de la práctica docente en la asignatura.

A partir de las ideas previas se pueden establecer conclusiones desde las creencias del alumnado, reflexionando de forma organizada sobre diferentes aspectos del proceso de enseñanza-aprendizaje, la actitud del profesorado ante la disciplina, su competencia, las estrategias empleadas para desarrollar el temario, pensando sobre la metodología, recursos, contenidos, valorando la consecución de objetivos....

En esta línea, tras la incorporación del Sistema Europeo de Transferencia de Créditos (ECTS), se está apostando por una formación centrada en el estudiante en la que prima un enfoque fundamentado en aprendizajes profundos orientados hacia el significado de lo que se desea aprender, en el que se relaciona el objeto de aprendizaje con los conocimientos previos y se integra la teoría con la práctica (Biggs, 2005; Biggs y Tang, 2007; Entwistle, 2007).

Una vía para lograrlo es la adopción de enfoques didácticos en la propia formación del profesorado que se aparten de los métodos tradicionales y se centren en la indagación y reflexión por parte de los estudiantes (Gil, 2017). 
Este enfoque conlleva modificaciones importantes en la labor docente, que en este nuevo marco de formación consiste en ofrecer una orientación y acompañamiento constante en los procesos reflexivos (Correa, 2015).

Este modelo aparece en discrepancia con los modelos tradicionales donde los aprendizajes son más superficiales (Gibbs, 1994). Teniendo en cuenta estas consideraciones de que la buena enseñanza resulta ser la que promueve el aprendizaje de los estudiantes (Ramsdem, 1992), consecuentemente la calidad de la enseñanza dependerá de la medida en que se percibe la necesidad de que el profesorado se comprometa a desarrollar su profesión sobre la base de nuevos roles que inciden directamente en las demandas surgidas a raíz de estos nuevos planteamientos de aprendizaje (De-Juanas y Beltrán, 2014: 62).

Estas demandas se concretan en la evaluación de aprendizajes, el desarrollo de nuevas herramientas para enseñar-aprender, la utilización de las nuevas tecnologías, la gestión de aprendizajes, la tutoría y la atención a la diversidad, entre otras (Haigh, 2010).

Por otro lado, existen algunas investigaciones que se centran en la identificación de las características del profesor eficaz desde la perspectiva de estudiantes universitarios o futuros profesores como la de Liu et al., (2015) donde se señalan las siguientes características: experto, paciente, atento, comprensivo, enseña bien, buenas habilidades comunicativas, hace cumplir las normas, motivador, organizado, buena personalidad, pasión por la enseñanza, construye relaciones, muestra respeto a los otros, equitativo, flexible, servicial, adecuada gestión del aula, creativo, divertido, sabe escuchar, logra la participación de los otros (alumnos, padres), amigable, propone desafíos a los estudiantes (con expectativas), utiliza diferentes modalidades.

Por todo ello, la autorreflexión se plantea como un enfoque a la enseñanza en la que el profesorado recoge datos sobre la enseñanza, examinan sus actitudes, creencias, suposiciones y prácticas de enseñanza y, utiliza la información obtenida como base para la reflexión crítica sobre la enseñanza (Richards y Lockhart, 1996:1) es un componente esencial en los programas de preparación de maestros debido a su potente impacto sobre las decisiones y la adopción de diferentes cursos de acción de los profesores (Carter, 2008), así como en el desarrollo de la capacidad de los profesores para dar un paso atrás y analizar la justificación de sus prácticas educativas (Loughran, 2011). Además, sirve como proceso de ayuda para profesores noveles, poniendo el énfasis en la autorreflexión para enseñar a otros (Ward y Mc-Cotter, 2004), el desarrollo de las posturas más críticas hacia la enseñanza (Bailey, 2006), reexaminar supuestos aceptados sobre la profesión (Richards y Lockhart, 1996), buscar prácticas más eficaces (Gun, 2011) y renovar su compromiso con el trabajo (Louis, Marks y Kruse, 1996). Es importante el dominio de la eficacia docente identificando aquellas dimensiones que pueden influir en la propia práctica, a pesar de la relevancia de este tema, estudios recientes han demostrado diversas limitaciones y la necesidad de realizar más investigaciones en este ámbito (Devine, Fahie, y McGillicuddy, 2013; Liu, Keeley, y Buskist, 2015; Meng et al., 2015). En suma, la autorreflexión es una potente herramienta para la preparación de los maestros debido a su impacto en tantas facetas de la profesión (Ramos, 2012). Estas herramientas ayuda a identificar aquellos aspectos donde se necesita más reflexión con el fin de promover los cambios oportunos (Martín, Prieto, y Lupión, 2014).

La investigación educativa ha comprobado que la percepción de los estudiantes sobre los distintos aspectos relacionados con los procesos de enseñanza aprendizaje que se van a seguir en las asignaturas juega un papel fundamental en el desarrollo de los mismos (Doménech y Fortea, 2015). 
Los datos que se recogen de las reflexiones iniciales pueden contribuir a conocer mejor las expectativas y demandas formativas de los futuros y futuras docentes, demandas que den respuestas a sus necesidades como profesionales de la educación y que los cualifique para realizar estas tareas dotándolos de herramientas necesarias que favorezca la reflexión sobre todos los aspectos que pueden ayudarles a mejorar el desarrollo de la profesionalidad (Serrano y Pontes, 2015).

\section{Método}

A pesar de que esta investigación sigue una metodología tipo encuesta, al utilizarse un cuestionario con preguntas abiertas, podemos considerarlo de naturaleza cualitativa (Buendía, Colás, y Hernández, 1998), descriptiva, basada en el propio trabajo, reflexionando el profesor/a y el grupo de alumnos/as siempre desde la perspectiva del pensamiento, basándose en la propia experiencia del alumnado que se forma.

\section{Objetivos}

El objetivo general de este trabajo es conocer las reflexiones iniciales del alumnado en cuanto a las expectativas de la asignatura de Métodos de Investigación y Diagnóstico en Educación social.

Como objetivos específicos se concretan los siguientes:

- Conocer las ideas previas del alumnado a la realización del curso.

- Conocer la reflexión del alumnado sobre el proceso de trabajo que se va a desarrollar en la asignatura.

- Conocer el pensamiento del alumnado sobre la actitud que esperan que debe tener el profesor/a.

\section{Muestra}

Por el tipo de estudio se trata de una muestra no probabilística de tipo intencional, formada por 94 alumnos y alumnas del Grado de Educación social de la Universidad de Huelva, adoptando como criterios de inclusión:

- Alumnado matriculado en la asignatura de Métodos de Investigación y Diagnóstico en Educación Social de la Universidad de Huelva.

- Turno de mañana y tarde.

- Asistencia regular. Concretamente el estudio se aplicó a una muestra de 59 alumnos que cursan el turno de mañana y 35 que cursan el turno de tarde. Esta cifra corresponde al total de número de estudiante que acudió el primer día de clase, procurando la espontaneidad de los comentarios y evitando indicaciones de la organización de la asignatura.

\section{Instrumentos y procedimiento de recogida de datos}

Para la recogida de datos se utiliza el documento de autorreflexión inicial (Ávila y Conde, 2013), se trata de un cuestionario formado por nueve ítems, 8 de ellos de preguntas abiertas y uno de pregunta cerrada, valorando del 1 al 5 el nivel de importancia referente a los objetivos planteados en la guía docente de la asignatura, siendo 1 nada importante y 5 muy importante. El cuestionario se elaboró tras establecer previamente un sistema de categorías que se presenta a continuación: 


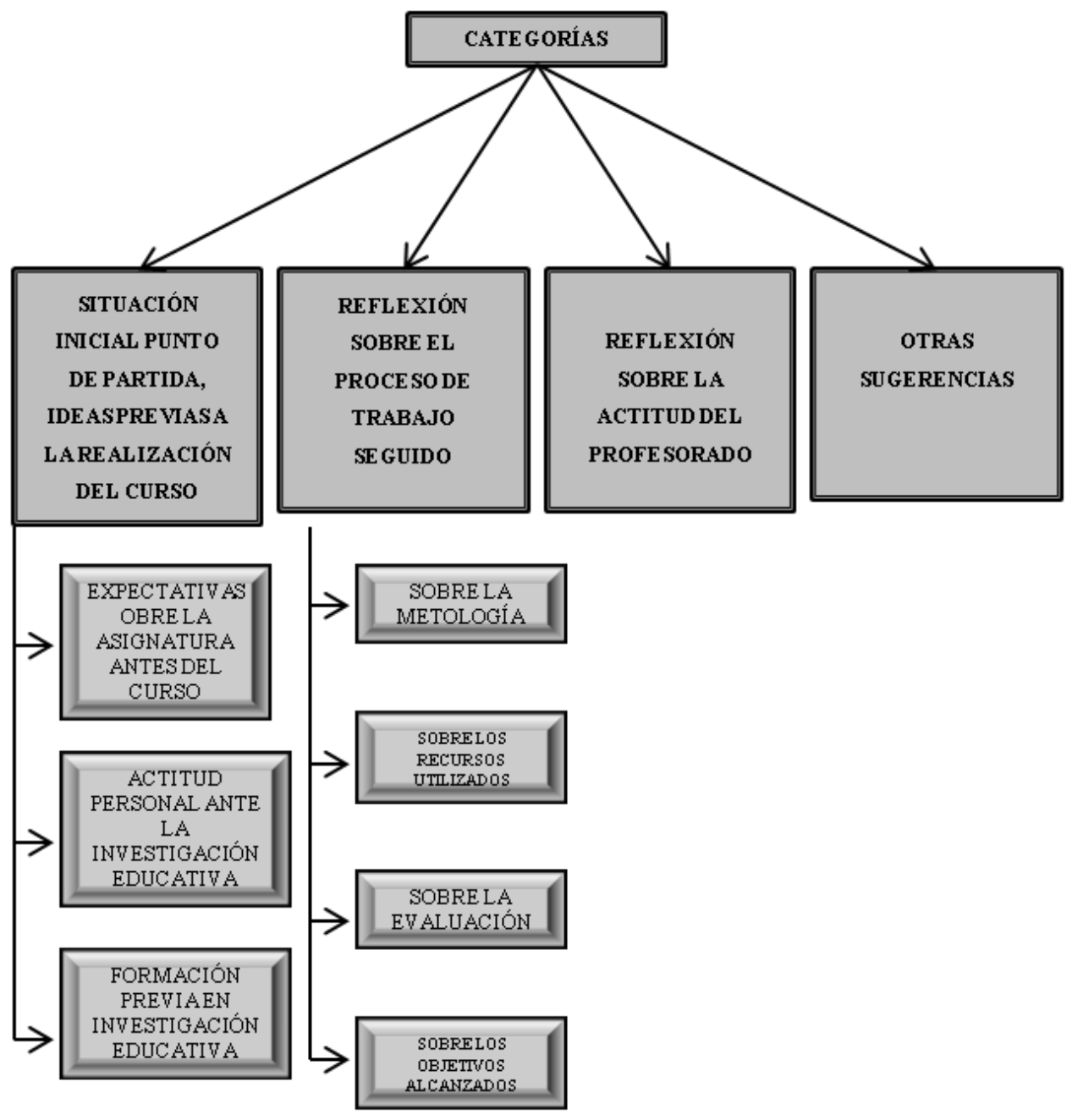

Figura 1. Sistema de categorías adaptado a la asignatura de Métodos de Investigación y Diagnóstico en Educación. Fuente: (Ávila y Conde, 2013)

Se denomina el documento "Autorreflexión Inicial" en el que se propone una reflexión para que se valore de manera personal las expectativas del curso que se va a compartir, indicando que las aportaciones serán tenidas en cuenta para la mejora de la práctica docente en la asignatura.

Se establece cuatro categorías:

- Situación inicial, punto de partida, ideas previas a la realización del curso.

- Reflexión sobre el proceso de trabajo.

- Reflexiones sobre la actitud del profesorado.

- Otras sugerencias.

Posteriormente se diversifica la información con diversas subcategorías en los dos primeros apartados. El esquema de trabajo se expresa en la figura 1.

Los ítems que se consideran en el cuestionario son: expectativas sobre la asignatura antes del curso, actitud personal ante la Investigación Educativa, formación previa en Investigación Educativa, reflexión sobre la metodología que se ha de seguir, reflexión sobre los recursos a utilizar, reflexión sobre la evaluación más idónea, valora la importancia de los 
objetivos que debería seguir la asignatura, aporta algunas reflexiones sobre la actitud que debe tener el profesor/a, y otras sugerencias.

Al tratarse de un cuestionario de preguntas abiertas no se aplicaron estadísticos de fiabilidad y validez, no obstante para la comprobación de la validez del instrumento se estableció dos momentos:

1. Validez teórica: se estableció un sistema de categorías tras la revisión de literatura y basándose en los estándares de la guía docente de la asignatura, una vez planteado el cuestionario se hizo una valoración de expertos pertenecientes al área de Métodos de Investigación y Diagnóstico en Educación (MIDE en adelante) de la Universidad de Huelva. El grupo de expertos estaba conformado por un total de cuatro personas, dos de ellos personal docente e investigador y otros dos profesionales de la orientación. Consideraron si las cuestiones proporcionaban información exacta y relevante, se revisó la selección y redacción de las mismas. Los cambios a destacar de la versión original del instrumento tras la revisión de expertos fueron: eliminación del primer ítem que hacía referencia a "¿por qué elegiste esta carrera?" no se consideró oportuno para el propósito del estudio y, reformulación del ítem referido a los "objetivos", en la versión facilitada al grupo de expertos, éste estaba formulado a través de una pregunta abierta, para que el alumnado planteara los objetivos que debería seguir la asignatura. Los expertos recomendaron que se incluyeran los objetivos establecidos en la guía docente de la asignatura y que el alumnado los valorase en una escala ad hoc siendo 1 nada importante y 5 muy importante.

2. Validez empírica: se realizó un análisis del contenido de las cuestiones planteadas. Obteniéndose a través del cuestionario datos sobre:

- las expectativas del alumnado antes del curso,

- actitud personal ante la investigación,

- formación previa del alumnado,

- expectativas sobre la metodología,

- expectativas sobre los recursos,

- expectativas sobre la Evaluación,

- nivel de importancia sobre los Objetivos alcanzados,

- pensamiento del alumnado sobre la actitud del profesorado.

Respecto a la fiabilidad, se comprobaron si las preguntas eran válidas para evaluar a distintos grupos de personas, para ello, las dimensiones propuestas se estudiaron analizando e interpretando la información contenida en el documento. Permitiendo la expresión cualitativa y matizada de la información obtenida (Aguaded y Tirado, 2008). Se realizó un estudio a partir de las evidencias recogidas en el trabajo de Ávila y Conde (2013) donde se utilizó el cuestionario en otra asignatura pertenecientes al área de MIDE.

El análisis de las diferentes categorías aplicadas en la otra asignatura reveló que eran muy similares en cuanto a su contenido.

El proceso de recogida de información se inicia el día de la presentación de la asignatura, procurando la espontaneidad de los comentarios y evitando indicaciones de la organización de la asignatura. 


\section{Análisis de datos}

Para el análisis de los datos se sigue cada uno de los elementos que se han determinado en el guión para el análisis cualitativo: transcripción y codificación, con ellos se consigue organizar toda la información obtenida en las distintas categorías y subcategorías facilitando así la descripción de datos agrupados con representaciones gráficas y la elaboración de las conclusiones del estudio.

A continuación, se muestra gráficamente una síntesis del proceso de análisis cualitativos, adaptación de Colás (1998).

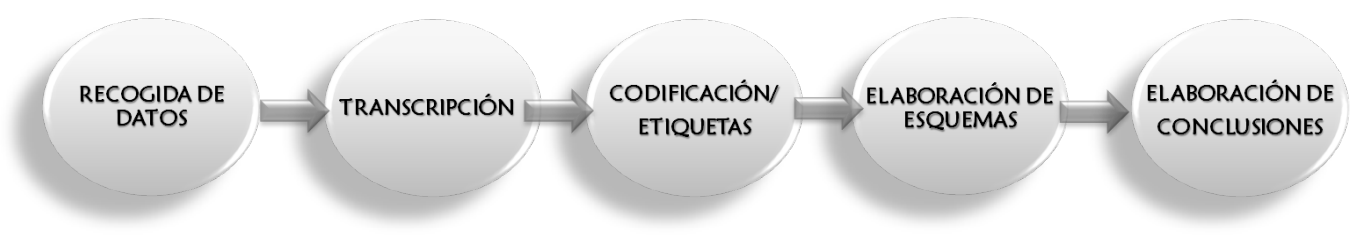

Figura 2. Proceso de análisis cualitativos. Fuente elaboración propia

De las aportaciones obtenidas en cada uno de los apartados se seleccionarán los elementos comunes expresándolos con una frase e incluyendo entre paréntesis el número de veces que se repite. En la valoración de los objetivos se sumarán los valores obtenidos por cada uno de ellos.

\section{Resultados}

A continuación se presentan los datos recogidos siguiendo el sistema de categorías aludido, haciendo coincidir aquellos que facilitan la tarea del análisis. Los datos se muestran a través de gráficos que ayudan a organizar la información más relevante, incluyendo entre paréntesis el número de veces que se repite. Seguidamente se realiza algunos comentarios descriptivos sobre ellos.

La figura 3 trata de las expectativas que el alumnado tiene sobre la asignatura de Métodos de Investigación y Diagnóstico en Educación Social (figura 3).

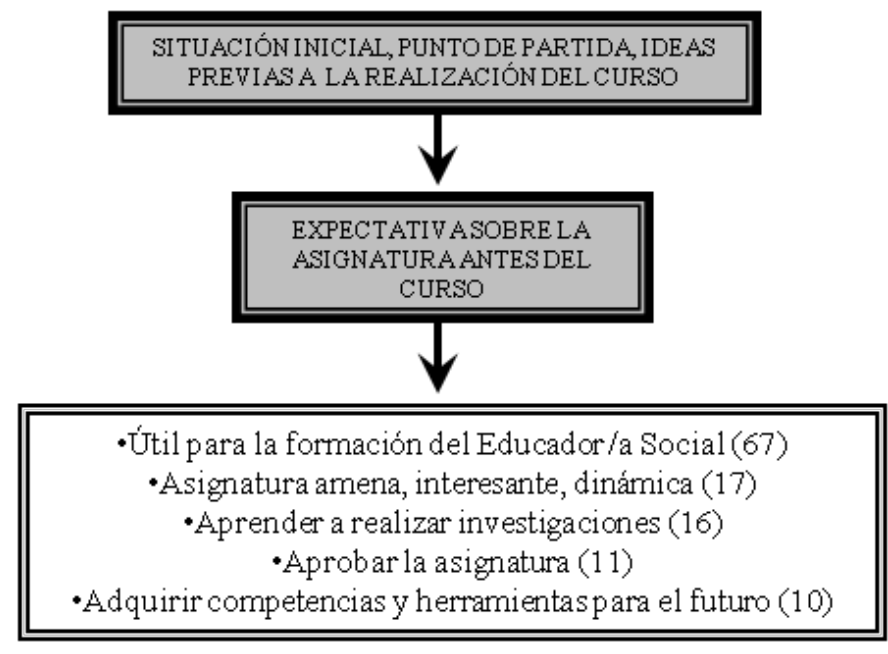

Figura 3. Información inicial sobre expectativas. Fuente: elaboración propia 
Como puede observarse, en la fase inicial el alumnado posiciona sus expectativas en el mismo nivel que cualquier otra asignatura que curse. Adquisición de conocimientos, que sea útil para su formación, que sea una asignatura amena, interesante, dinámica, que le permita realizar investigaciones, aprobar la asignatura y que les sirva para adquirir las herramientas necesarias para poder desempeñar las funciones como educadores/as sociales en un futuro. Estas respuestas, son las más habituales.

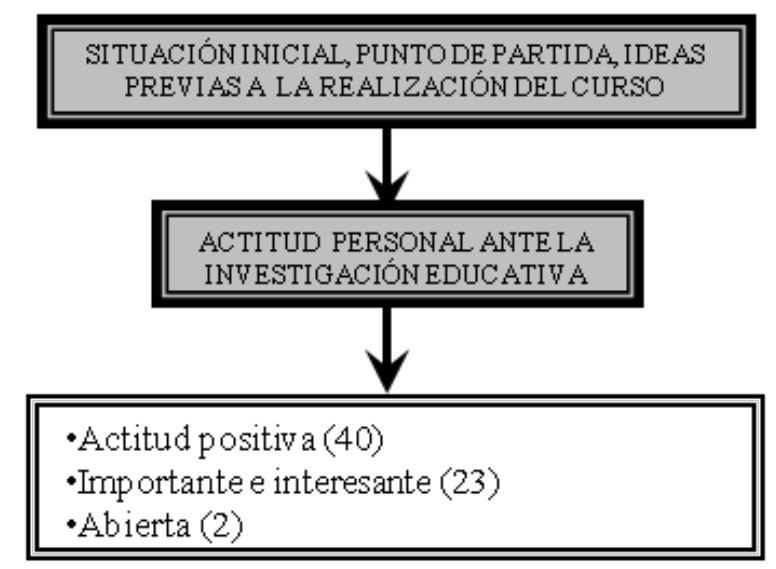

Figura 4. Información inicial sobre actitud. Fuente: elaboración propia

En los datos recogidos respecto a la actitud personal ante la investigación educativa el alumnado, en general, muestra una actitud positiva ante el aprendizaje de esta asignatura, reflejando una actitud reflexiva y de utilidad hacia la misma. Por otro lado, un grupo de ellos aseguran su posicionamiento con alusiones relativas a la importancia e interés de la asignatura, expresando en sus comentarios palabras que denotan cierta curiosidad e interés "estoy interesado y motivado". Además se refleja también una actitud abierta y dispuesta al trabajo.

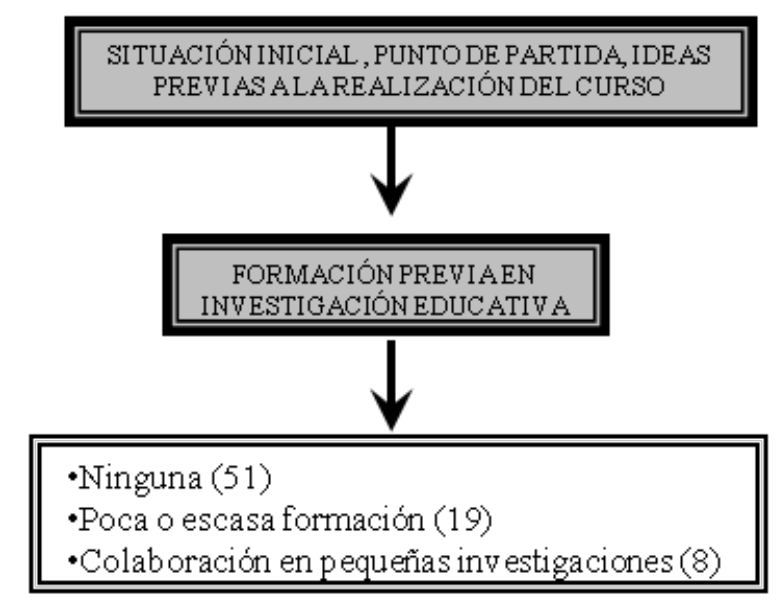

Figura 5. Formación previa en Investigación educativa. Fuente: elaboración propia

El punto de partida es previsible, al acceder al alumnado sin formación preliminar. Se trata de un campo que aún desconocen o del que tienen poca experiencia (figura 5). La mayoría de la muestra no tiene formación previa en investigación o la que tienen es muy básica, tan sólo 8 personas hacen alusión de haber colaborado en pequeñas labores de investigación. 
El segundo bloque que se presenta a continuación, hace referencia al proceso de trabajo que se espera seguir en la asignatura. En la figura 6 , se recoge las principales alusiones realizadas sobre la metodología.

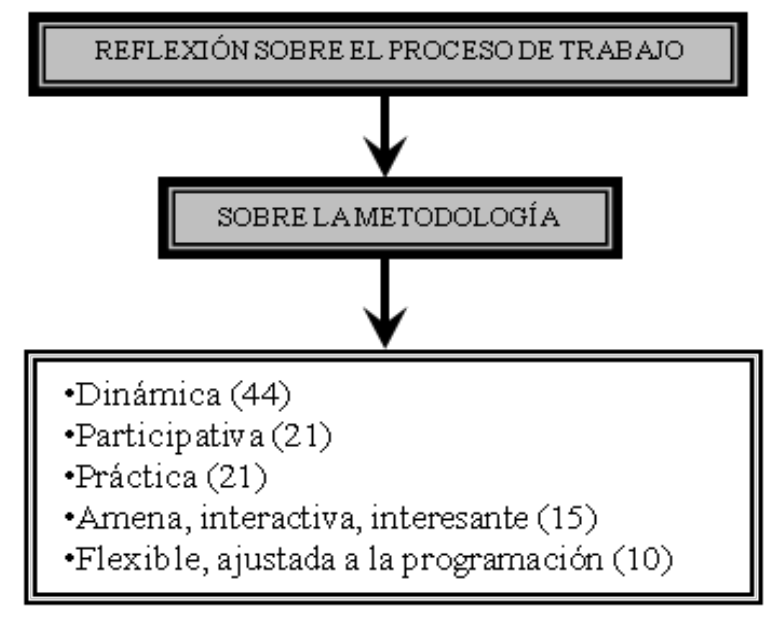

Figura 6. Información inicial sobre metodología. Fuente: elaboración propia

En general, el alumnado demanda una metodología dinámica, participativa, práctica, amena, flexible. Parece defenderse un proceso de trabajo fundamentalmente práctico que permita la participación y un mayor nivel de protagonismo del alumnado en el aula.

En la figura 7 se presenta los datos relacionados con los recursos esperados:

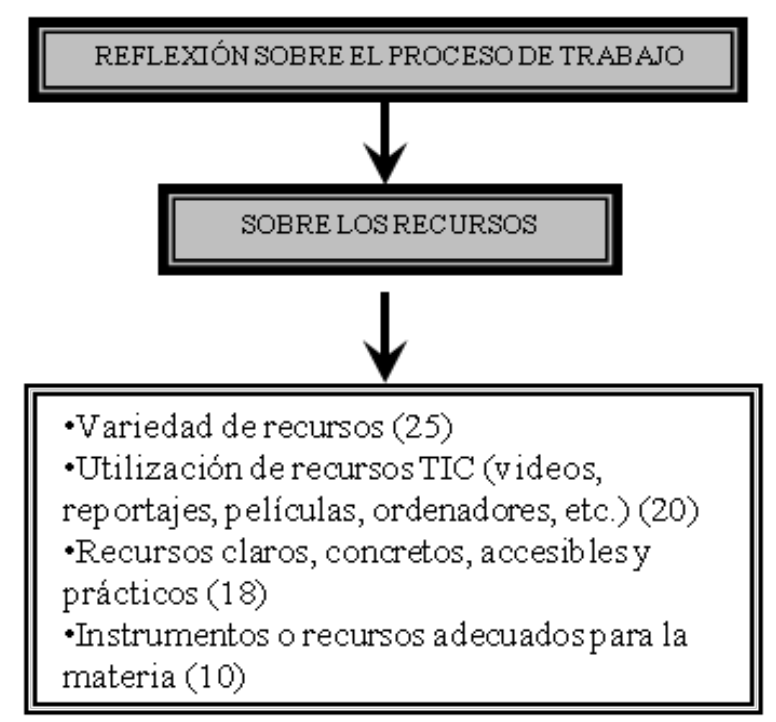

Figura 7. Información inicial sobre recursos. Fuente: elaboración propia

En este apartado se destaca el deseo de utilizar variedad de recursos tales como ordenadores, diapositivas, pizarras, proyectores, espacios informáticos, materiales necesarios para las exposiciones de los temas, etc. Muy presente esta en los comentarios del alumnado el utilizar las TIC en el ámbito escolar. También se hace mención a la utilización de recursos claros, concretos, accesibles y prácticos en distintas aportaciones 
que hace el alumnado al respecto "Recursos que nos ayuden en nuestro futuro trabajo como educadores/as sociales".

\section{REFLEXIÓN SOBRE EL PROCESODE TRABAJO}

SOBRE LAEVALUACIÓN

-Evaluación justa y objetiva (34)

-Evaluar todo el trabajo y el esfuerzo (21)

- Continúa (15)

Figura 8. Información inicial sobre la evaluación. Fuente: elaboración propia

Seguidamente, se reflexiona sobre la evaluación, la principal aportación que se hace al respecto es que se lleve a cabo una evaluación justa y objetiva, sobre todo se hace referencia a las calificaciones. Por otro lado se insiste en evaluar todo el trabajo y el esfuerzo, subrayando el deseo de que el mayor peso de las calificaciones recaiga en este último y no en pruebas escritas tales como exámenes, test, etc. Por otro lado también se señala la importancia de una evaluación continua y sin demasiadas dificultades.

Respecto a los objetivo que debería cubrir esta asignatura, este apartado, se analiza de forma cuantitativa, el alumnado valora cada uno de los objetivo, atendiendo a su nivel de preocupación, del 1 al 5, siendo 1 el menor nivel de importancia y 5 el máximo.

En la siguiente gráfica se representa el nivel de importancia de cada uno de los objetivos propuestos. El resultado que se expresa en la grafica es la suma de los valores obtenidos en cada uno de ellos.

Elaborar un informe de investigación

Saber analizar e interpretar los datos obtenidos en los diferentes diseños de investigación

Seleccionar, elaborar y utilizar distintos instrumentos y técnicas de recogida de datos

Diseñar y realizar investigaciones sencillas con modelos adecuados al problema planteado

Especificar las realizaciones y hallazgos más relevantes en cada enfoque

Conocer los enfoques básicos de investigación educativa

Diferenciar las fases principales a través de las que se desarrolla un proceso de investigación en

Introducir al alumnado en el lenguaje y conceptos básicos de la investigación científica

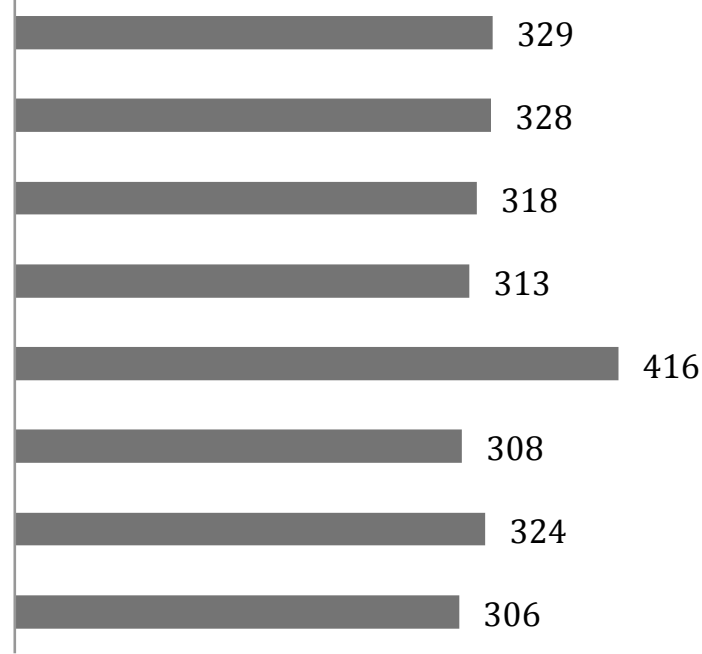

Gráfico 1. Información inicial sobre objetivos. Fuente: elaboración propia 
En general, como puede observarse en el gráfico 1, todos los encuestados manifiestan un gran nivel de preocupación e importancia por cada uno de los objetivos planteados, no obstante los que más se destacan son: especificar las realizaciones y hallazgos más relevantes en cada enfoque; elaborar un informe de investigación; saber analizar e interpretar los datos obtenidos en los diferentes diseños de investigación y diferenciar las fases principales a través de las que se desarrolla un proceso de investigación en educación.

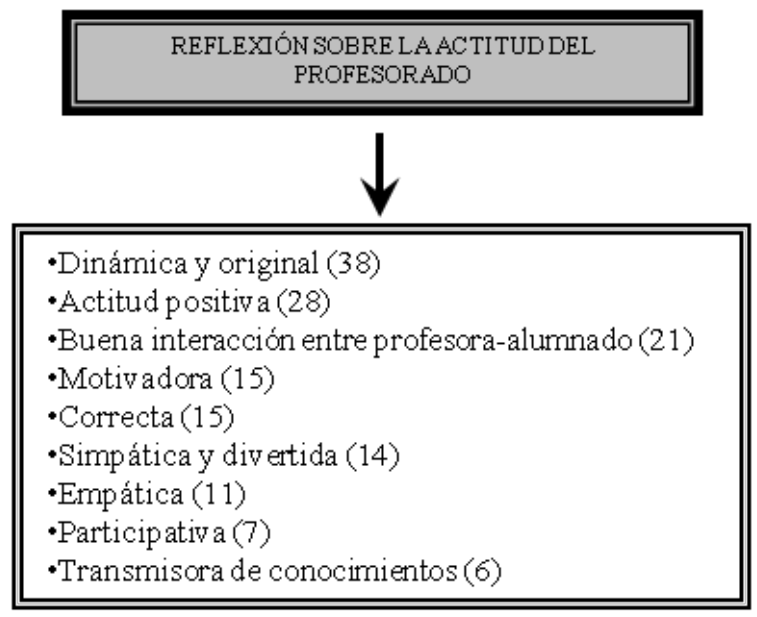

Figura 9. Reflexiones iniciales sobre el profesorado. Fuente elaboración propia

Respecto a los datos recogidos sobre la actitud (figura 9) que debería tener el profesorado, parece que el alumnado tiene unas ideas claras de cómo debe ser el perfil de un buen docente, o al menos, las que más se ajustan a sus creencias: dinámico/a, original, divertido/a, una actitud positiva, buena interacción entre profesorado-alumnado, motivador/a, simpático/a, participativo/a, etc.

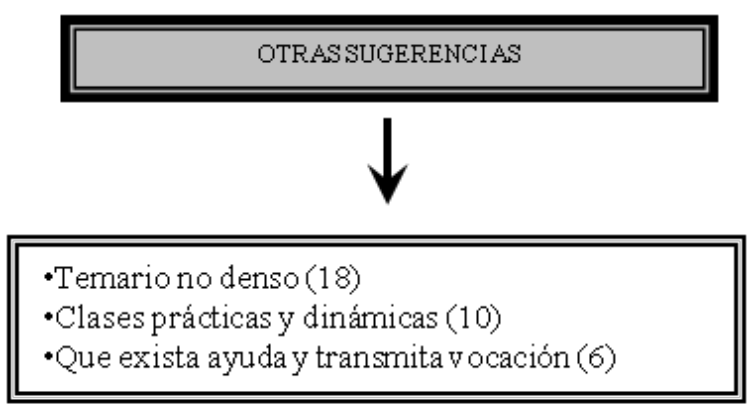

Figura 10. Información sobre otras sugerencias. Fuente: elaboración propia

La última categoría que incluye el cuestionario se dedica a otras sugerencias donde el alumnado puede plantear aquellas propuestas que quiera hacerle llegar al docente y que no estén contempladas en los apartados anteriores, a pesar de que la mayoría de los encuestados no contestan este apartado, cabe destacar las siguientes sugerencias: que exista ayuda y transmita vocación; temario no denso y clases prácticas y dinámicas. 


\section{Discusión y conclusiones}

El objetivo de esta investigación ha sido aportar una herramienta de autorreflexión inicial que ayude al profesorado a reflexionar sobre su práctica docente y explorar las valoraciones que los estudiantes hacen al respecto. Pensamos que con esta herramienta proporcionamos una manera de promover la reflexión del profesorado para mejorar su práctica profesional (Larrievee, 2008; Mena, Sánchez y Tillema, 2011 y Zhu, 2011). En este sentido, de acuerdo con Ramos (2012), la autorreflexión debería ser un componente esencial de los contenidos de las materias estudiadas. Por otro lado, sirve como un proceso de ayuda para el profesorado, reflexionando sobre su práctica docente, buscando métodos más eficaces y significativos e incorporando a su práctica aquellos aspectos que parecen favorecer los procesos de enseñanza aprendizaje en el aula (Bailey, 2006; Gun, 2011; Ward y Mc-Cotter, 2004). Los resultados que se obtienen de esas reflexiones ayudan a identificar aspectos donde se necesitan más meditación con el fin de promover los cambios oportunos (Doménech y Fortea, 2015).

Los aspectos abordados representan parcelas claves de la enseñanza y el aprendizaje en la actualidad (Martín, Prieto y Lupión, 2014). En este sentido se precisa aceptar un enfoque más práctico adecuado a problemas reales a lo que los alumnos tendrán que afrontar (Gil, 2017). De acuerdo con Martín, Prieto y Jiménez (2015) se hace relevante situar al alumnado como protagonista de su aprendizaje.

Del análisis de las valoraciones realizadas por el alumnado sobre la práctica docente, se coincide con el modelo aportado por Beltrán y Pérez (2005) y De-Juanas y Beltrán (2014), en el que el estudio de las diferentes propuestas obtenidas en cada categoría permiten afirmar que dichas propuestas se pueden integrar y sintetizar en:

- Competencia académica: dominio de la materia por parte del profesorado y habilidades que permitan construir aprendizajes.

- Profesorado facilitador de aprendizajes: empleo de diversos instrumentos, recursos, empleo de metodologías dinámicas, participativas, prácticas, amenas, flexibles.

- Competencia pedagógica: capacidad didáctica que permita el desarrollo de competencias y valores en los alumnos.

- Personalidad del profesorado: comprende su capacidad de optimismo, liderazgo, entusiasmo, interacción con el alumnado, extroversión, solidaridad, etc.

- Eficiencia personal: el profesorado permite al alumnado solucionar problemas de manera creativa asumiendo el rol de experto.

Por otro lado respecto a las características que tiene que tener el docente, se coincide con las aportadas en otros estudios como los de Koutrouba (2012), Meng, Muñoz y Wu (2015) y Peng et al. (2014), donde a través de autoencuestas con preguntas abiertas, entrevistas u observaciones directas en el aula, han ido elaborando listas con factores que contribuyen a la eficacia docente. Destacan las aportaciones de Miller (2012), quien identifica diez características propias del buen profesor: entusiasta, creativo, con sentido del humor, que desafía a los alumnos, alentador, paciente, que no se da por vencido, se interesa por los estudiantes, conoce bien la gramática, disponible para atender a los alumnos, que trate bien, sea justo, y que deje su bagaje emocional fuera del aula; organizadas en cuatro áreas: características afectivas, habilidades (creatividad, abierto a nuevos desafíos), técnicas de gestión del aula y conocimientos académicos. 
Tras la aplicación de esta herramienta de autorreflexión inicial se extraen las siguientes conclusiones:

- Se comprueba que es abundante tanto en calidad como en cantidad la información que aporta este tipo de herramientas ya que el alumnado tiene oportunidad de expresar de una forma abierta sus reflexiones acerca de distintos aspectos relacionados con los procesos de enseñanza-aprendizaje y que consideran importantes a la hora del desarrollo de una asignatura.

- Las propuestas que en él se recogen pueden servir de guía al docente a la hora de diseñar sus clases y elaborar la metodología más adecuada y eficaz. En este sentido la utilización de este instrumento en el contexto universitario puede conllevar implicaciones prácticas para incluir en la formación permanente del profesorado universitario competencias sociales y emocionales (asertivo, empático), como aquellas psicodidácticas que promueven la motivación y disciplina en el aula (atento, flexible, participativo, orientador, líder).

- Permite conocer algunas expectativas, rasgos y deseos de su alumnado con referencia al aprendizaje que espera realizar. En este sentido, se espera que sea de utilidad para su futuro laboral, que sepan realizar investigaciones y adquirir competencias y herramientas para el futuro. Por lo tanto, sin perder la óptica de una buena teoría que fundamente los procesos prácticos, los conocimientos que se desarrollan en esta asignatura en el grado de educación social, deberían contar con más situaciones reales que aporten un enfoque más profesional y de inmediata aplicación práctica, como pueden ser el trabajo de campo en centros educativos donde el alumnado tenga posibilidad de observar escenarios de trabajos y realizar investigaciones reales que pongan en juego las competencias adquiridas.

- Permite conocer la predisposición con la que parte el alumnado que va a cursar la asignatura específica. Si tienen actitud positiva o negativa, si la consideran importante, interesante. Estos indicadores orienta a los docentes a ofrecer una formación no sólo teórico/práctica sino también actitudinal, ayudando a valorar y reconocer la importancia que, en este caso, la investigación socioeducativa tiene en la labor del educador/a social.

- Permite identificar aquello que motiva al alumnado. Utilización de recursos diversos, actividades prácticas enfrentando al alumnado a situaciones reales de trabajo, clases dinámicas donde el alumnado adquiera protagonismo y no sólo sea un mero receptor de conocimientos. Generando en el aula un buen clima de trabajo con profesorado empático, cercano, flexible, que ayude a motivar al alumnado que transmita vocación y que ayude y guíe al alumnado a lo largo de todo el proceso.

En consecuencia, es esencial entrenar al profesorado desde la universidad en la adquisición de adecuadas habilidades sociales y estrategias que favorezcan esta relación con el alumno en su formación (Reoyo, Carbonero, Martín, 2017).

En general, la aplicación de este tipo de herramientas supone una doble vertiente en la formación permanente del profesorado: fomentar la investigación educativa y contribuir a la mejora de la práctica docente. Desde este trabajo se pretende ver la necesidad de utilizar una herramienta para la reflexión inicial que ayude a la mejora de la práctica docente, y contribuya a conocer las expectativas y demandas formativas del alumnado dando respuestas a sus necesidades como futuros profesionales de la educación. 


\section{Referencias}

Aguaded, J.I. y Tirado, R. (2008). Los centros TIC y sus repercusiones didácticas en primaria y secundaria en Andalucía. Educar, 41, 61-90.

Ávila, J. y Conde, S. (2013). Modificación del alumnado que cursa Tutoría y Orientación en Educación Infantil. Tendencias Pedagógicas, 22, 211-228.

Bailey, K.M. (2006). Language teacher supervision. Cambridge: Cambridge University Press.

Biggs, J. (2005). Calidad del aprendizaje universitario. Madrid: Narcea.

Biggs, J. y Tang, C. (2007). Teaching for quality learning at university: what the student does (3nd ed.). Phildelphia, Pa.: Society for Research into Higher Education. Open University Press.

Buendía, L., Colás, P. y Hernández, F. (1998). Métodos de Investigación en Psicopedagogía. Madrid: McGraw-Hill.

Carter, B.A. (2008). Teacher-learners' voices: Not the same old song. Innovation in Language Learning and Teaching, 2(1), 33-46.

Correa, E. (2015). La alternancia en la formación inicial docente: vía de profesionalización. Educar, 51(2), 259-275. doi:10.5565/rev/educar.712.

De-Juanas, A. y Beltrán, J. (2014). Valoraciones de los estudiantes de ciencias de la educación sobre la calidad. 17(1), 59-82.

Devine, D., Fahie, D. y McGillicuddy, D. (2013). What is 'good' teaching? Teacher beliefs and practices about their teaching. Irish Educational Studies, 32, 83-108. doi:10.1080/03323315.2013.773228.

Doménech, F. y Fortea, M.A. (2015). Validez Factorial de un Cuestionario para Evaluar la Percepción Inicial de los Estudiantes Universitarios sobre la Evaluación de los Aprendizajes. Electronic Journal of research in Educational Psychology, 13(3), 569-586.

Entwistle, N. (2007). Research into student learning and university teaching. BJEP Monograph Series II, Student Learning and University Teaching, 1, 1-18.

Gibbs, G. (Ed.) (1994). Improving Student Learning. Theory and Practice. Oxford: The Oxford Centre for Staff Development.

Gil, J. (2017). Rasgos del profesorado asociados al uso de diferentes estrategias metodológicas en las clases de ciencias. Enseñanza de las Ciencias, 35(1), 175-192.

Gun, B. (2011). Quality self-reflection through reflection training. ELT Journal, 65(2) 126-135.

Haigh, A. (2010). Enseñar bien es un arte. Madrid: Narcea.

Imbernón, F. (Coord) (2002). La investigación educativa como herramienta de formación del profesorado. Reflexión y experiencias de investigación. Barcelona: GRAÓ.

Koutrouba, K. (2012). A profile of the effective teacher: Greek secondary education teachers' perceptions. European Journal of Teacher Education, 35(3), 359-374. doi:10.1080/02619768.2011.654332.

Larrivee, B. (2008). Development of a tool to assess teachers' level of reflective practice. Reflective Practice, 9, 341-360. 
Liu, S., Keeley, J. y Buskist, W. (2015). Chinese College Students' Perceptions of Characteristics of Excellent Teachers. Teaching of Psychology, 42(1), 83-86. doi:10.1177/0098628314562684.

Loughran, J. (2011). On becoming a teacher educator. Journal of Education for Teaching, 37(3), 279-291.

Louis, K.S., Marks, H.M. y Kruse, S. (1996). Teachers' professional community in restructuring schools. American Educational Research Journal, 33(4), 757-798.

Ramsden, P. (1992). Learning to teach in higher education. London: Routledge.

Martín, C., Prieto, T. y Jiménez, A. (2015). Tendencias del profesorado de ciencias en formación inicial sobre las estrategias metodológicas en la enseñanza de las ciencias. Estudio de un caso en Málaga. Enseñanza de las Ciencias, 33(1), 167-184.

Martín, C. Prieto, T. y Lupión, T. (2014). Profesorado de ciencias en formación inicial ante la enseñanza y el aprendizaje de las ciencias: ¿perfil innovador o tradicional? Revista Electrónica Interuniversitaria de Formación del Profesorado, 17(1), 149-163.

Mena, J., Sánchez, E. y Tillema, H. (2011). Promoting teacher reflection: what is said to be done. Journal of Education for Teaching, 37, 21-36.

Meng, L., Muñoz, M.A. y Wu, D. (2015). Teachers' perceptions of effective teaching: a theory-based exploratory study of teachers from China. Educational Psychology, (ahead-of-print), 1-20. doi:10.1080/01443410.2015.1008402.

Peng, W.J., McNess, E., Thomas, S., Wu, X.R., Zhang, C., Li, J.Z. y Tian, H.S. (2014). Emerging perceptions of teacher quality and teacher development in china. International Journal of Educational Development, 34(1), 77-89. doi:10.1016/j.ijedudev.2013.04.005.

Ramos, F. (2012). Patroneando un barco a la deriva: Mejorando la preparación de los maestros de los colegios católicos durante el primer año a través de la autorreflexión. Estudios Sobre Educación. ESE, 22, 73-91.

Reoyo, N., Carbonero, M.Á., Martín, L.J. (2017) Características de eficacia docente desde las perspectivas del profesorado y futuro profesorado de Secundaria. Revista de Educación, 376, 62-96.

Richards, J.C. y Lockhart, C. (1996). Reflective Teaching in Second Language Classrooms. New York: Cambridge University Press.

Serrano, R. y Pontes, A. (2015). Expectativas ante la formación inicial entre el alumnado del Máster de Profesorado de Enseñanza Secundaria. Revista de Investigación Educativa, 33(2), 489-505. DOI: http://dx.doi.org/10.6018/rie.33.2.203471.

Ward, J.R. y Mccotter, S.S. (2004). Reflection as a visible outcome for preservice teachers. Teaching and Teacher Education, 20(3), 243-257.

Zhu, X. (2011). Student teachers' reflection during practicum: plenty on action, few in action. Reflective Practice, 12, 763-775. 